\title{
Comparison of Hansenula wingei, a Petite-negative, Obligately Aerobic Yeast, to the Petite-positive Yeast Saccharomyces cerevisiae
}

\author{
By MARJORIE CRANDALL \\ Deportment of Microbiology, Indiana University, Bloomington, Indiana, 4740I, U.S.A.
}

(Received Io October 1972; revised 20 November 1972)

\begin{abstract}
SUMMARY
After extensive ethidium bromide (EB) treatment or ultraviolet irradiation no petite mutants could be isolated from the obligate aerobe Hansenula wingei. Petitenegativity is correlated with a lack of glucose repression. Hansenula winge $i$ is sensitive to EB on fermentable as well as non-fermentable carbon sources. Growth arrest by EB of single cells on agar is immediate but one or two divisions occur in liquid before the sensitive cells stop growing. Although EB treatment of the sensitive wild-type does not result in either petite mutagenesis or cell killing, there is a reversible inhibition of growth observed when EB-treated cells are plated on nonfermentable substrates. Mutants resistant to EB were isolated on either glucose + EB or glycerol +EB plates. Mutants resistant to EB in glycerol medium are also resistant to $\mathrm{EB}$ in glucose medium. However, mutants isolated on glucose + EB medium are still sensitive to EB in glycerol medium. When mutants resistant to EB in glycerol medium revert to sensitivity they retain resistance to EB in glucose medium. Resistance to EB in glucose medium is recessive in the diploid.
\end{abstract}

\section{INTRODUCTION}

It is the purpose of this paper to compare petite-production in the well-studied yeast Saccharomyces cerevisiae to the petite-negative yeast Hansenula wingei. These species names are abbreviated $S c$ and $H w$.

Hansenula wingei $(\mathrm{Hw})$ has two interesting properties which make it a valuable object for the study of mitochondrial inheritance: it is sexually agglutinative and it is an obligate aerobe (Wickerham, 1970). The ability of the haploids to agglutinate promotes conjugation. Typically $80 \%$ of the cells will mate in $5 \mathrm{~h}$ in a glucose, magnesium-phosphate buffer which does not support growth (Brock, I96I; Crandall \& Brock, I968). This property would facilitate the study of transmission and recombination of mitochondrial genes during zygote formation and subsequent diploid growth. Because $\mathrm{Hw}$ cannot grow fermentatively, it is completely dependent on mitochondrial function, as are higher organisms. Information gained from the study of mitochondrial function in a micro-organism such as $H w$ may be more relevant to mammalian systems than studies of facultatively anaerobic yeasts such as $S c$.

The best studied mitochondrial mutation in yeast is the petite $(\rho-)$ or cytoplasmic respiratory-deficient mutation in $S c$ (recent references which review the literature on the petite mutation include: Kellerman, Biggs \& Linnane (I969), Coen et al. (I970), Nagley \& Linnane (1970), Bolotin et al. (I97I), Goldring, Grossman \& Marmur (197I), Luha, Sarcoe \& Whittaker (I97I), Mahler \& Perlman (I97I), Perlman \& Mahler (I97I $a, b$ ), Preer (197I), Weislogel \& Butow (I97I) and Mahler \& Perlman (I972)).

Any mutation which destroys mitochondrial function in $\mathrm{Hw}$ should be lethal since even 
fermentable substrates such as glucose are respired. It was the main objective of this investigation to look for petite mutants in $H w$ and to isolate other types of mutants that would be valuable in studying mitochondrial function in this obligately aerobic organism. Agents used for attempted mitochondrial mutagenesis in $H w$ were u.v. irradiation and EB which is highly efficient in causing the petite mutation in the mitochondrial genome of $S c$ (Slonimski, Perrodin \& Croft, 1968). Studies of mutants of $H w$ isolated after u.v. mutagenesis or EB selection are described in Crandall (1973).

\section{METHODS}

Media. Experimental growth conditions and media were those of Coen et al. (I970). Natural media contained: I \% yeast extract (Difco Laboratories, Detroit, Michigan, U.S.A.); I \% Bacto-peptone (Difco); 0.05\% M-Na, K-phosphate buffer, pH 6.25 (Sörensen); 2.3\% Bacto-agar (Difco); and 2\% carbon sources: glucose (G) or glycerol (GLY). Differential medium contained $0.1 \% \mathrm{G}+2 \% \mathrm{GLY}$. On this medium petites or $\rho^{-}$give rise to small colonies, whereas the normal grande or $\rho^{+}$give rise to large colonies. Natural medium for $\mathrm{G}$ broth and $\mathrm{G}$ slants did not contain buffer and was supplemented with $20 \mu \mathrm{g} / \mathrm{ml}$ adenine. EB dissolved in sterile water was added to $50 \mu \mathrm{M}(20 \mu \mathrm{g} / \mathrm{ml})$ final concentration to G or GLY natural medium just before pouring plates $(\mathrm{G}+\mathrm{EB}$ or $\mathrm{GLY}+\mathrm{EB})$. The EB medium was protected against light. Actidione (cycloheximide) dissolved in sterile water was added to $20 \mu \mathrm{g} / \mathrm{ml}$ final concentration to $\mathrm{G}$ natural medium just before pouring plates $(\mathrm{G}+\mathrm{ACT})$. Glucose minimal medium was yeast nitrogen base without amino acids (Difco) to which was added $2 \% \mathrm{G}$ and $2.3 \%$ Bacto-agar (Difco). Minimal medium was autoclaved as the complete medium for $20 \mathrm{~min}$ at I IO ${ }^{\circ} \mathrm{C}$. Required supplements were added at $20 \mu \mathrm{g} / \mathrm{ml} \mathrm{just}$ before pouring plates. Stock cultures of $\mathrm{Hw}$ on natural $\mathrm{G}$ medium slants grew well but lost viability within 2 to 3 months so stock slants were prepared using medium previously reported for $\mathrm{Hw}$ (Crandall \& Brock, I968). This medium (YG) contains: $0.7 \%$ yeast extract (Difco) $3 \%$ glucose; $0.5 \% \mathrm{KH}_{2} \mathrm{PO}_{4}$; and $2 \%$ Bacto-agar (Difco). Stock cultures of $\mathrm{Hw}$ on YG slants have, in the past, remained viable for up to a year or longer (M. Crandall, observation). In some experiments this medium was used with different carbon sources: ethanol (YE), sodium lactate (YL) or glycerol (YGLY). Liquid YG medium is $\mathrm{pH} 5 \cdot 0$.

Culture conditions. Liquid precultures were inoculated from $\mathrm{G}$ slants to $5 \mathrm{ml}$ of $\mathrm{G}$ broth/ tube. Cells were aerated on a reciprocating shaker during overnight growth at $28{ }^{\circ} \mathrm{C}$.

Yeast strains. Strains of $S c$ used as controls were grandes of complementary mating type and complementary auxotrophic requirements: IL 8-4D $\alpha$ ura and ILI66-6D $a$ his. The diploid hybrid $(a / \alpha)$ of these two strains is a prototroph. The petite mutant tested was IL $8-8 \mathrm{C} \mathrm{R} 5 / 3$ $\alpha$ his trp.

Strains of $H w$ used were $5 \mathrm{cyh}$ lys and 21 ade. The origin of these haploid mutants is as follows: diploid NRRL Y2340 (a poor sporulator) was isolated by L. J. Wickerham from the frass of bark beetles living in conifer trees in the western U.S. From one tetrad of y2340, two ascospore cultures, 5-9A and 2I-9D, of opposite mating type were isolated by A. I. Herman. Wild-type strain 5-9A is of agglutination and mating type 5. A spontaneous mutant, $5 \mathrm{cyh}$, resistant to $100 \mu \mathrm{g} / \mathrm{ml}$ of Actidione (cycloheximide) was selected and then mutagenized with nitrous acid using the procedure of A. I. Herman (personal communication). A lysine-requiring auxotroph, $5 \mathrm{cyh} l y s$, was thus obtained. From a population of 5 cyh lys mutagenized with u.v. were obtained two types of EB-resistant mutants, those resistant to $20 \mu \mathrm{g} \mathrm{EB} / \mathrm{ml}$ on $\mathrm{G}$, e.g. 5 cyh lys etb-36, and those resistant to $20 \mu \mathrm{g} \mathrm{EB} / \mathrm{ml}$ on GLY, e.g. 5 cyh lys etb-6r. Wild-type strain $2 \mathrm{I}-9 \mathrm{D}$ is of agglutination and mating type $2 I$. 
A leaky auxotroph requiring adenine, $2 I$ ade, which grows slowly on minimal and turns red was a gift from A. I. Herman. Strain $2 I$ ade was mutagenized with u.v. to obtain two mutants, $2 I$ ade his, an auxotroph requiring both adenine and histidine and $2 I$ ade glp, a leaky glycerol-negative mutant (Crandall, 1973). Haploids VIA and VIB are wild-type ascospore isolates of opposite mating type dissected by A. I. Herman from one tetrad of NRRL YB-4662, a good sporulating diploid isolated from the eastern U.S. by L. J. Wickerham. Strain VIA agglutinates and mates with type 5 and strain VIB agglutinates and mates with type $2 I$.

Preservation of stock cultures using silica gel. The method of Fink (unpublished) was employed. Silica gel (Type I, chromatographic grade, 60-200 mesh, cat. no. S7375, Sigma Chemical Co., St Louis, Missouri, U.S.A.) was used to half-fill vials (dram, $15 \times 60 \mathrm{~mm}$ borosilicate glass). The caps (rubber lined) were autoclaved then dried overnight. The vials, without caps, were dry-sterilized for $3 \mathrm{~h}$ at $80{ }^{\circ} \mathrm{C}$. The vials were tightly capped immediately after they cooled and stored in a freezer until used. Yeast strains were streaked on G plates and incubated 2 days at $28{ }^{\circ} \mathrm{C}$. Vials at freezer temperatures were placed in holes of a metal block also chilled in the freezer. Cells were scraped off agar plates using a small metal spatula sterilized by alcohol and flaming. The yeast cells were mixed well with the silica gel, the caps tightened and the vials returned to the chilled metal block since heat is given off during the drying process. Silica gel stocks can be stored in the refrigerator or at room temperature and should remain viable for 5 years or longer. Cultures can be revived by sprinkling small portions of the dried cells on $\mathrm{G}$ plates and incubating 2 days at 28 to $30{ }^{\circ} \mathrm{C}$.

Chemicals. Ethidium bromide (2,7-diamino-1o-ethyl-9-phenyl-phenanthridinium bromide) was a gift from Boots Pure Drug Co. Ltd, Nottingham or was purchased from Calbiochem, La Jolla, California, U.S.A. (B grade) or from Sigma Chemical Co. Actidione (cycloheximide) was purchased from Upjohn Co., Kalamazoo, Michigan, U.S.A., or from Sigma Chemical Co. All other chemicals were of reagent grade purity.

Ultraviolet mutagenesis. Liquid cultures in G medium of each haploid ( $5 \mathrm{cyhlys}$ and $2 \mathrm{I}$ ade) were harvested, washed twice in sterile saline $(0.88 \% \mathrm{NaCl}$ solution), and then resuspended at about $10^{7}$ cells $/ \mathrm{ml}$. Samples of $10 \mathrm{ml}$ suspension in Petri plates were irradiated at $\mathrm{I} 6$ ergs $/ \mathrm{mm}^{2} / \mathrm{s}$ for 60 and $100 \mathrm{~s}$ which gave 25 and $4 \%$ survival respectively. Similar survival percentages are obtained with $S c$ under these conditions of irradiation (B. Dujon, personal communication). The irradiated suspensions were harvested and resuspended in $5 \mathrm{ml} \mathrm{G}$ broth for overnight growth and phenotypic expression.

\section{RESULTS}

Search for spontaneous petites in $H w$. No spontaneous petite mutants were present in populations of strains 5 cyh lys or strain 21 ade. Small colonies on differential medium were found to be glycerol-positive upon replica plating. In contrast, $S c$ yields several percent (strain dependent) spontaneous petite mutants on differential plates.

Sensitivity to EB during growth of wild-type $H w$ on solid medium. Growth of both $S c$ and $H w$ was completely inhibited on GLY + EB plates since GLY can only be oxidized aerobically and EB inhibits mitochondrial respiratory enzyme synthesis. EB added at $20 \mu \mathrm{g} / \mathrm{ml}$ to $\mathrm{G}$ plates completely inhibited growth of $H w$ without affecting the efficiency of plating (e.o.p.) of either the $\rho^{+}$or $\rho^{-}$strain of $S c$ on G (Table I). However, under these conditions $100 \%$ of the $S c$ colonies are petites. Sensitivity of $H w$ to EB in G medium is understandable since $H w$ cannot ferment (Wickerham, I970) and requires full respiratory enzyme potential even for the oxidation of $\mathrm{G}$. Although there is no petite mutagenesis in $\mathrm{Hw}$, there is some 
effect produced by EB. If EB is present at borderline inhibitory concentrations ${ }^{-}(4 \mu \mathrm{g} / \mathrm{ml})$ during growth on $\mathrm{G}$ the cytochrome spectrum shows partial petite character in that cytochromes $a+a_{3}$ and $b$ are lower and cytochrome $c$ is higher. The same concentration of EB $(4 \mu \mathrm{g} / \mathrm{ml})$ in GLY medium completely inhibits growth.

Sensitivity to $E B$ during growth of wild-type $H w$ in $G+E B$ broth. Results from plate studies showing sensitivity to $20 \mu \mathrm{g} \mathrm{EB} / \mathrm{ml}$ in G medium and borderline inhibition at $4 \mu \mathrm{g} / \mathrm{ml}$ were confirmed in studies of liquid cultures. After the addition of EB to an exponential culture of 5 cyh lys growing on $\mathrm{G}$, this sensitive culture completed one doubling before growth was arrested (Fig. I). Mahler \& Perlman (I97I) found similar results in that cells of $H w$ managed but one mass doubling in the presence of $\mathrm{EB}(20 \mu \mathrm{g} / \mathrm{ml}$ in $5 \% \mathrm{G}$ medium $)$ after $7.5 \mathrm{~h}$. In the experiment of Fig. I, there was no loss in viability after $7.5 \mathrm{~h}$ at $20 \mu \mathrm{g} / \mathrm{ml}$. There was, however, some loss of viability after $7.5 \mathrm{~h}$ in the presence of $100 \mu \mathrm{g} / \mathrm{ml}$. After $7.5 \mathrm{~h}$, in the presence of $20 \mu \mathrm{g} \mathrm{EB} / \mathrm{ml}$ there were no resistant mutants present in this sensitive population $(<\mathrm{I}$ in 14000$)$.

In this same experiment the turbidity of the $20 \mu \mathrm{g} / \mathrm{ml}$ culture increased fourfold as compared to only a twofold increase in viable counts before growth stopped. In a duplicate experiment of the EB-sensitive wild-type growing in $\mathrm{G}$ broth $+20 \mu \mathrm{g} \mathrm{EB} / \mathrm{ml}$ it was again found that the turbidity increased several-fold more than the viable count. It is not known whether this disparity is due to dry-weight increases without cell division or whether a division is occurring but the daughter cell is non-viable. Ephrussi \& Hottinguer (I95I) reported that during petite mutagenesis of $S c$ with acriflavin only the bud is converted into a petite, the mother cell remains grande.

Growth inhibition caused by previous growth in the presence of EB. EB has a reversible effect on $H w$. When the cells from the experiment of Fig. I were plated on different carbon sources it was found that, as a function of time in the presence of $20 \mu \mathrm{g} \mathrm{EB} / \mathrm{ml}$, there was a decrease in the e.o.p. of 5 cyh lys on lactate (Fig. I) and an increase in the percentage of small colonies on all carbon sources (Fig. 2). On G plates the percentage of small colonies was least. The small colonies appeared after a growth lag of several days. If the plates had not been incubated for a long time the small colonies would not have developed and it would have appeared that killing by EB had occurred. Replica plating of the $G$ plates for each kinetic sample showed that all the cells were still capable of growing on all carbon sources but evidently growth was more inhibited on ethanol, glycerol and lactate than on a fermentable substrate (glucose) after EB treatment.

Lack of microcolony development. Bulder $(1964 b)$ reported that certain petite-negative yeasts will produce microcolonies on acriflavin medium. These microcolonies lost viability upon subculturing and therefore might be lethal petite mutants. No such microcolonies were observed after cells of the EB sensitive wild-type ( 5 cyh lys) were plated on G medium containing either 20 or $100 \mu \mathrm{g} \mathrm{EB} / \mathrm{ml}$. Only single cells were found (very rarely doublets) indicating immediate growth inhibition after plating on EB.

Sensitivity to EB under non-growing conditions. Strain 5 cyh lys was treated with $20 \mu \mathrm{g} / \mathrm{ml}$ of $\mathrm{EB}$ in liquid YG or YGLY growth medium at a growth-restricting temperature $\left(45^{\circ} \mathrm{C}\right)$ and then plated on G or GLY or differential medium at time intervals up to $4 \mathrm{I} \mathrm{min}$. The conditions used by Perlman \& Mahler (I97I $a$ ) to induce petites in $S c$ were duplicated so that strict comparison of the results could be made. There was no loss of viability and no petites produced in $\mathrm{Hw}$ by this rigorous $\mathrm{EB}$ treatment at $45{ }^{\circ} \mathrm{C}$ which produced up to $100 \%$ petites in $S c$ in less than $5 \mathrm{~min}$. Even though no petite mutagenesis occurred there was, however, some effect. The cells become increasingly growth-inhibited on GLY as a function of time of treatment with EB. This was true whether the cells were treated in $\mathrm{G}+\mathrm{EB}$ or 


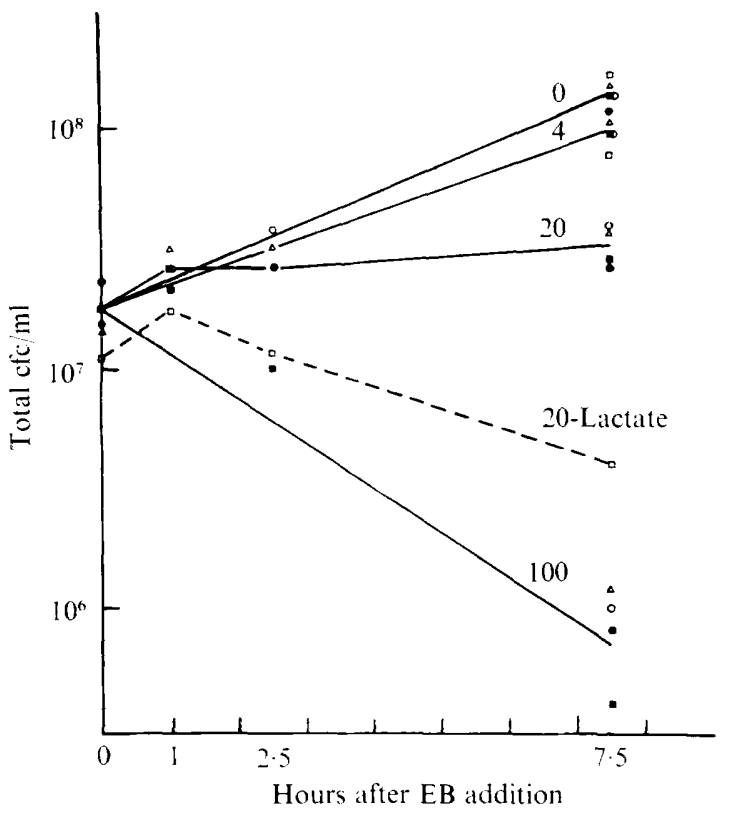

Fig. 1. Sensitivity to EB during growth of wild-type $H w$ in $\mathrm{G}+\mathrm{EB}$ broth. Strain 5 cyh lys was grown overnight in natural $\mathrm{G}$ broth containing $0.05 \mathrm{M}$-Sörensen phosphate buffer, pH 6.2 , then diluted about $1 / 100$ into fresh medium. The cultures were aerated in a $30{ }^{\circ} \mathrm{C}$ water bath with bubble tubes and sterile Antifoam 'A' (Dow Corning Corporation, Midland, Michigan, U.S.A.) was added when necessary. When the culture reached exponential phase it was divided into four culture flasks and different amounts of EB were added from a freshly prepared stock solution at $10 \mathrm{mg} / \mathrm{ml}$. Final concentrations of EB were $0,4,20$ and $100 \mu \mathrm{g} / \mathrm{ml}$. Samples for viable counts were diluted in $0.88 \%$ saline and samples of 0.1 containing 100 to 400 cells were spread on six kinds of media (YG, GLY, YE, YL, Y differential and natural G $+20 \mu \mathrm{g} \mathrm{EB} / \mathrm{ml}$ plates). Colonies were counted after I week at which time small colonies had appeared on some plates. Total colony counts (small plus large) are expressed as total cells forming colonies $(\mathrm{cfc}) / \mathrm{ml}$.

GLY + EB broth and was demonstrated by two criteria: (i) the e.o.p. on GLY as compared to $\mathrm{G}$ or differential medium decreased to about 0.6 after $4 \mathrm{I} \mathrm{min}$; and (ii) the percentage of small colonies on differential medium increased with time of EB treatment. This inhibition of growth on GLY was reversible by growth on G as shown by two criteria: (i) the e.o.p. of $\mathrm{G} /$ differential was about $\mathrm{I} \cdot 0$; and (ii) all 39 small colonies from differential medium were glycerol-positive when later transferred to GLY plates.

Ultraviolet mutagenesis. After irradiation and phenotypic expression, cells were serially diluted and spread on G plates. These were replica plated to GLY plates. In this mutant population from $2 I$ ade there was I \% auxotrophs after irradiation to $4 \%$ survival. From this same population was isolated only one mutant with an impairment in growth on GLY (2I ade glp). This same treatment of $S c$ would have yielded a majority percentage of petites. Mutant $2 I$ ade glp is not a petite as judged by the criteria presented in Crandall (1973). This same mutant ( $2 I$ ade $g l p$ ) also changes the frequency of diploid mitotic segregation of a gene for EB resistance (Crandall \& Richter, to be published).

Petite-negativity in $\mathrm{Hw}$ is correlated with lack of glucose repression. When $\mathrm{G}$ was added to a final concentration of $5 \%$ to cultures of $H w$ or $S c$ growing on GLY, Sc immediately slowed to a much decreased growth rate but $H w$ maintained its exponential growth rate. Inhibition of the $S c$ culture lasted $40 \mathrm{~min}$ in duplicate experiments and then the culture 


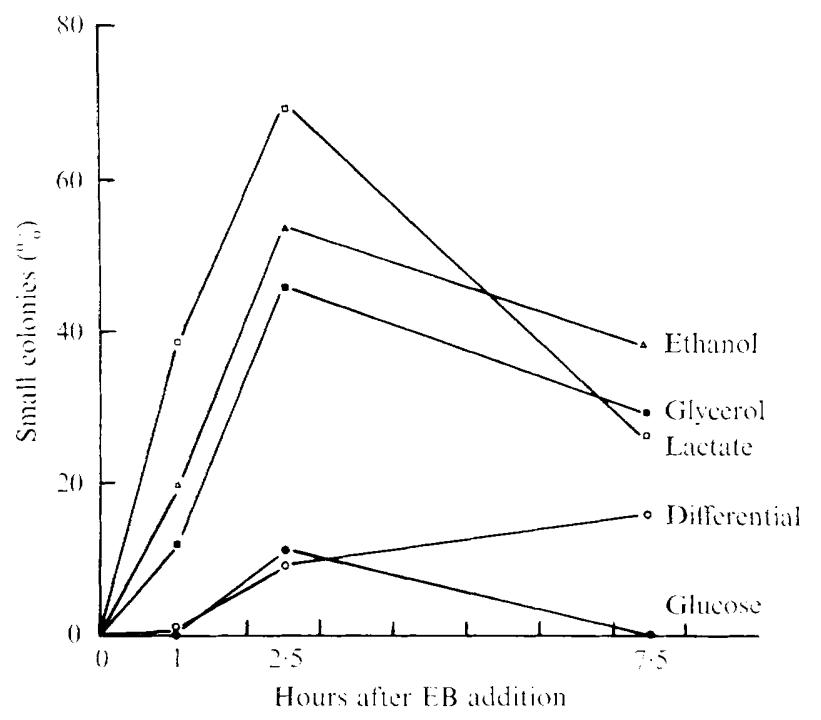

Fig. 2. Growth inhibition caused by previous growth in the presence of EB. Same experiment as Fig. I. The culture of $5 \mathrm{cyhlys}$, containing $20 \mu \mathrm{g} \mathrm{EB} / \mathrm{ml}$, was diluted and spread on plates containing different carbon sources. After I week incubation small and large colonies were counted. Small colonies $\times 100$ were divided by the total number of colonies to obtain $\%$ small colonies.

recommenced exponential growth at an enhanced rate, presumably now growing fermentatively on the added G. This glucose repression of respiratory enzyme synthesis in $S c$ is the 'contre-effect Pasteur' described by Slonimski (I956). The 40 min lag probably represents the time during which inducible enzymes for glucose fermentation are being synthesized. The immediate response of $S c$ to added $G$ shows that glucose repression must also act via a constitutive mechanism, perhaps by competition with GLY for an intermediate. It is clear that neither aspect of glucose repression is present in $H w$. Other observations are in agreement with the conclusion that $H w$ lacks glucose repression. Cells grown on $2 \% \mathrm{G}$ plates have cytochrome spectra essentially identical to cytochrome spectra from derepressed cells of $S c$ (Crandall, I973). Cells grown in $3 \% \mathrm{G}$ broth for electron microscopic studies of conjugation showed well-developed mitochondria (Conti \& Brock, I965).

Isolation of EB-resistant mutants. Since $H w$ was found to be sensitive to EB on fermentable as well as non-fermentable carbon sources it was decided to isolate mutants resistant to EB either in G or GLY medium. After u.v. mutagenesis and expression, cells were diluted and spread on G +EB plates. To obtain mutants resistant to EB in GLY medium, cells were first grown on G during expression and then in GLY medium. They were then concentrated $\times I 0$ and $O \cdot I \mathrm{ml}$ was spread on GLY + EB plates. The frequency of mutants resistant to $\mathrm{EB}$ in $\mathrm{G}$ medium in the mutagenized population was about $1 \mathrm{O}^{-4}$ and the frequency of mutants resistant to EB in GLY medium was about $10^{-6}$. Both frequencies were increased about 100-fold by u.v. mutagenesis.

Mutants isolated on G +EB plates were sensitive on GLY + EB plates. Mutants isolated on GLY + EB were cross-resistant to EB in G medium. There were stable and unstable mutants in both classes. Several isolates from GLY + EB medium when cultured on nonselective medium reverted to EB sensitivity on GLY + EB plates but retained EB resistance 


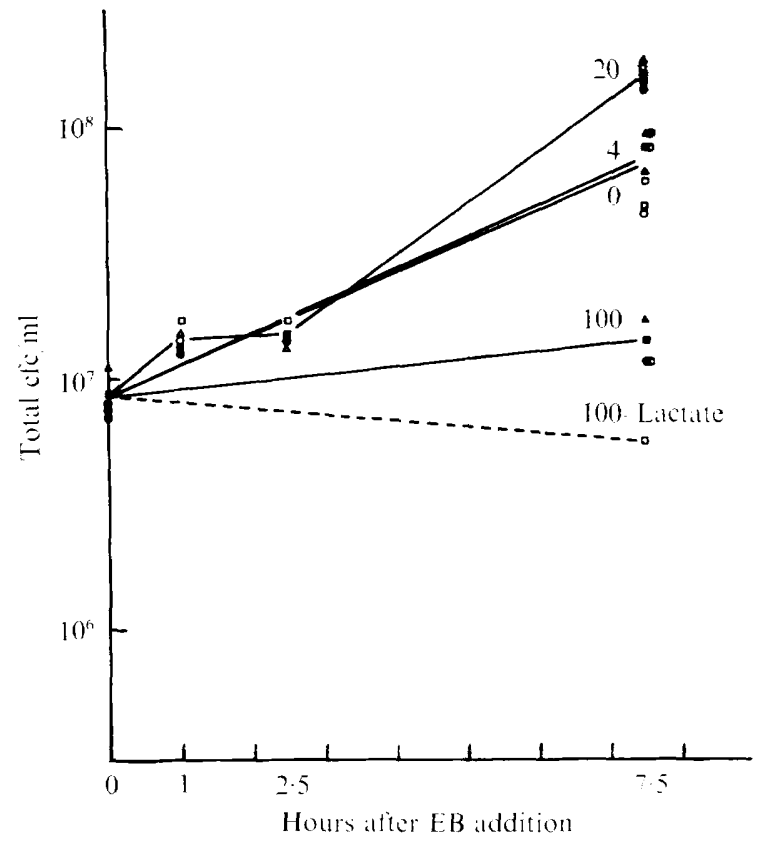

Fig. 3. Resistance to EB during growth of $e t b-36$ in $\mathrm{G}+\mathrm{EB}$ broth. Strain 5 cyh lys etb-36 was grown and tested for EB resistance as described in the legend of Fig. I.

Species

Mating types

Conjugation

Oxygen requirement for growth

Petite mutation

Glucose repression

Strains

Growth on agar plates
Glucose
Glycerol
Glucose + EB
Glycerol + EB

Reversion to wild-type
Table I. Comparison of two yeats
Saccharomyces cerevisiae

$$
a \text { and } \alpha
$$

The strains below are non-agglutinative

$$
\text { I to } 10 \% \text { zygotes in growth }
$$
medium

Facultative aerobe: both fermentation and respiration

Positive

Positive

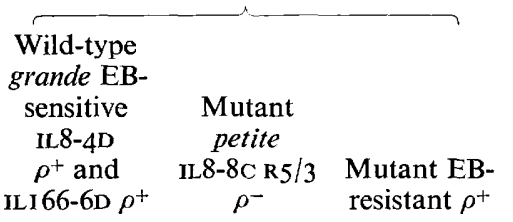

$$
\begin{aligned}
& ++ \\
& ++ \\
& ++
\end{aligned}
$$$$
-
$$$$
++
$$

Wild-type Never reverts$$
\text { Unstable }
$$

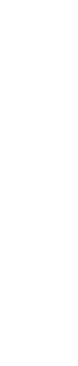

\section{Hansenula wingei}

Strains 5 and $2 I$ Sex-specific agglutination $80 \%$ zygotes in buffer

\section{Obligate aerobe:} only respiration

Negative

Negative

in $\mathrm{G}$ medium. These observations indicated that these mutants are different and that in order for a cell to grow on GLY $+\mathrm{EB}$, it must have both resistance mutations. This conclusion is consistent with the frequencies of mutation for each type of EB-resistant mutant 


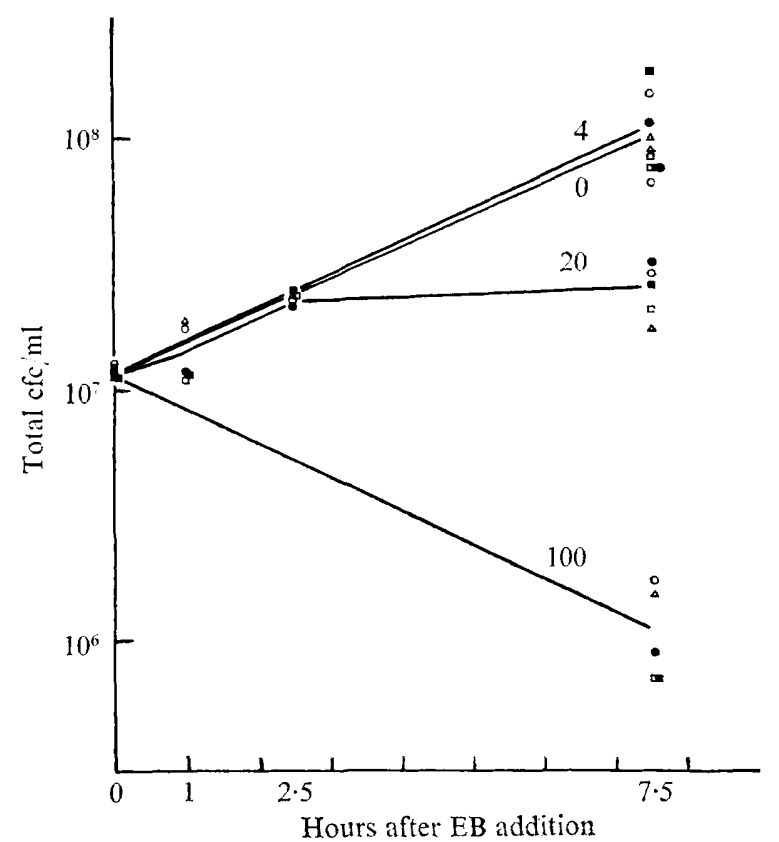

Fig. 4. Sensitivity to EB during growth of the heterozygous diploid etb-36/+ in G+EB broth. Diploid strain 5 cyh lys etb-36 $\times 21$ ade his was grown and tested for EB sensitivity as described in the legend of Fig. I.

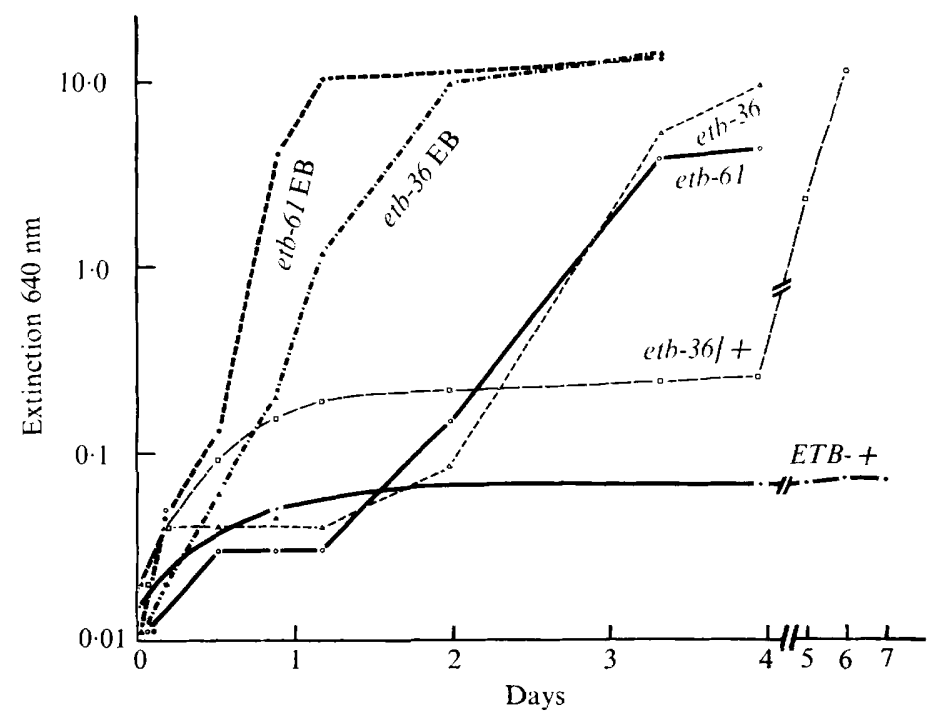

Fig. 5. Comparison of growth of EB-sensitive and EB-resistant strains in $\mathrm{G}+\mathrm{EB}$ broth. Strains $5 c y h$ $\mid y s, 5$ cyh lys $e t b-36,5$ cyh lys etb-6t and the diploid $e t b-36 \mid+$ (VIA) were pregrown in natural G broth medium with $0.05 \mathrm{M}$-Sörensen phosphate buffer, $\mathrm{pH} 6 \cdot 2$, for 2 days. Since the EB resistance mutants had a lag period of several days in EB broth it was necessary to prepare EB precultures of each mutant. Preculture $e t b-36 \mathrm{~EB}$ was grown to stationary phase in $\mathrm{G}+\mathrm{EB}$ broth, then on G+EB plates and then in $G+E B$ broth for 2 days. Preculture $e t b-6 I$ EB was grown to stationary phase in GLY + EB broth, then on GLY + EB plates and then in G +EB broth for 2 days. All six precultures were diluted $\mathrm{r} / 500$ into $\mathrm{G}+\mathrm{EB}$ broth. Growth was followed by measuring the extinction at $640 \mathrm{~nm}$ in side-arm Erlenmeyer flasks in a 'Spectronic 20' spectrophotometer (Bausch Lomb, Rochester, New York, U.S.A.). Above an extinction of 0.6 , the culture was diluted before measurement. 


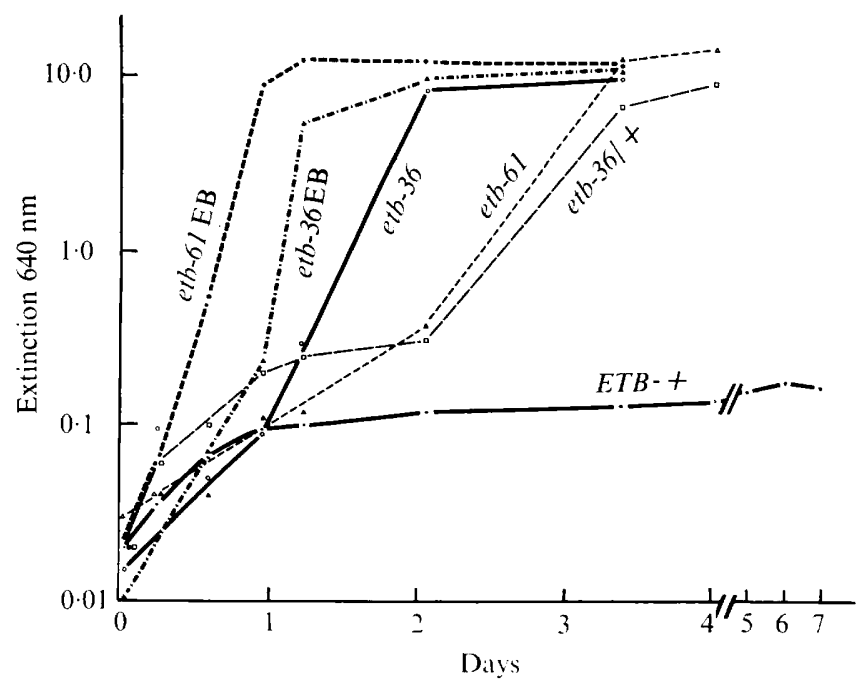

Fig. 6. Comparison of growth of EB-sensitive and EB-resistant strains in GLY + EB broth. Same strains and experimental design as Fig. 5 except GLY was used as carbon source. Mutant $e t b-36$ EB was precultured in $\mathrm{G}+\mathrm{EB}$ broth, then on $\mathrm{G}+\mathrm{EB}$ plates, and then in GLY +EB broth for 2 days. Mutant $e t b-6 I$ EB was precultured in GLY + EB broth, then on GLY + EB plates, and then in GLY + EB broth for 2 days.

and with the fact that higher concentrations of EB are required to inhibit growth on $\mathrm{G}$ as compared to GLY. None of these EB-resistant mutants are petites since they were all glycerol-positive. Stable mutants were cultured on non-selective medium and when replica plated to EB medium, I00 \% of the cells were resistant. Growth in the presence and absence of EB for $S c$ and $H w$ parental and mutant strains is summarized in Table I.

Resistance to $E B$ during growth of etb-36 in $G+E B$ broth. In the same kinetic experiment represented in Fig. I and 2, mutant etb-36 was compared to its EB-sensitive parent. It is clear from viable counts on five different media that this mutant grows as well in the presence of $20 \mu \mathrm{g} \mathrm{EB} / \mathrm{ml}$ as in its absence (Fig. 3). This mutant was isolated on G plates containing this same concentration of EB and therefore it was of interest to see if it was resistant to higher EB concentrations. Fig. 3 shows that although this mutant is sensitive to $100 \mu \mathrm{g} \mathrm{EB} / \mathrm{ml}$ it is resistant to the loss in viability observed with the EB-sensitive culture at $100 \mu \mathrm{g} / \mathrm{ml}$.

Sensitivity to $E B$ during growth of the heterozygous diploid etb-36/+in $G+E B$ broth. In the same kinetic experiment represented in Fig. I to 3 it was found that the resistance to EB in glucose medium is recessive. The same kinetics of growth inhibition were obtained for the $e t b-36 /+$ heterozygote as for the EB-sensitive wild-type haploid (compare Fig. I, 4) including little or no inhibition at $4 \mu \mathrm{g} / \mathrm{ml}$, one doubling in the presence of $20 \mu \mathrm{g} / \mathrm{ml}$ and some loss of viability at $100 \mu \mathrm{g} / \mathrm{ml}$ after $7.5 \mathrm{~h}$. The only minor difference between the heterozygote and the haploid was no decrease in the e.o.p. of the diploid on lactate after EB treatment but this may be because the diploid has twice the gene dosage for enzymes required for lactate utilization. The fact that sensitivity is dominant is very important for the interpretation of the results of the genetic analysis of this nuclear mutation presented in Crandall \& Richter (1973). Since sensitivity is dominant, the correct gene symbol for the wild-type is $E T B-+$.

Comparison of growth of EB-sensitive and EB-resistant strains in $G+E B$ or $G L Y+E B$ 
Table 2. Distinction between EB adaptation or genetic selection

Selective cultures grown in G + EB were from the experiment of Fig. 5 and 6 . The diploid which grew in $G+E B$ broth was diluted $\mathrm{I} / \mathrm{I} 00$ into $\mathrm{G}+\mathrm{EB}$ and allowed to grow to stationary phase again in selective broth. These same cultures, grown for many generations in selective media, were 'deadapted' by diluting $\mathrm{I} / 100$ into non-selective $\mathrm{G}$ broth growing 2 days, diluting again $\mathrm{I} / \mathrm{roo}$ into $\mathrm{G}$ broth and growing another 2 days. Selective, 'deadapted' and non-selective broth cultures of each strain were diluted in saline and $0.1 \mathrm{ml}$ containing 50 to 200 cells was spread on $\mathrm{G}$ plates in duplicate and also on $\mathrm{G}+\mathrm{EB}$ and GLY $+\mathrm{EB}$ plates. In addition, $\mathrm{O} \cdot \mathrm{I} \mathrm{ml}$ containing approximately $10^{4}$ cells was spread on G+EB and GLY + EB plates. After 2 days of growth the G plates were counted and one of each duplicate was replica plated to both $G+E B$ and GLY $+E B$ plates. The culture of $E B T$ - + indicated to have grown in $\mathrm{G}+\mathrm{EB}$ was the sensitive control of Fig. 5. It did not grow for 7 days in $\mathrm{G}+\mathrm{EB}$ broth but after 8 days it started to grow and by 9 days had reached an extinction of $\sim$ Io equal to full stationary phase. It can be seen this culture is still sensitive on GLY +EB plates but now contains a small percentage of mutants resistant on $\mathrm{G}+\mathrm{EB}$.

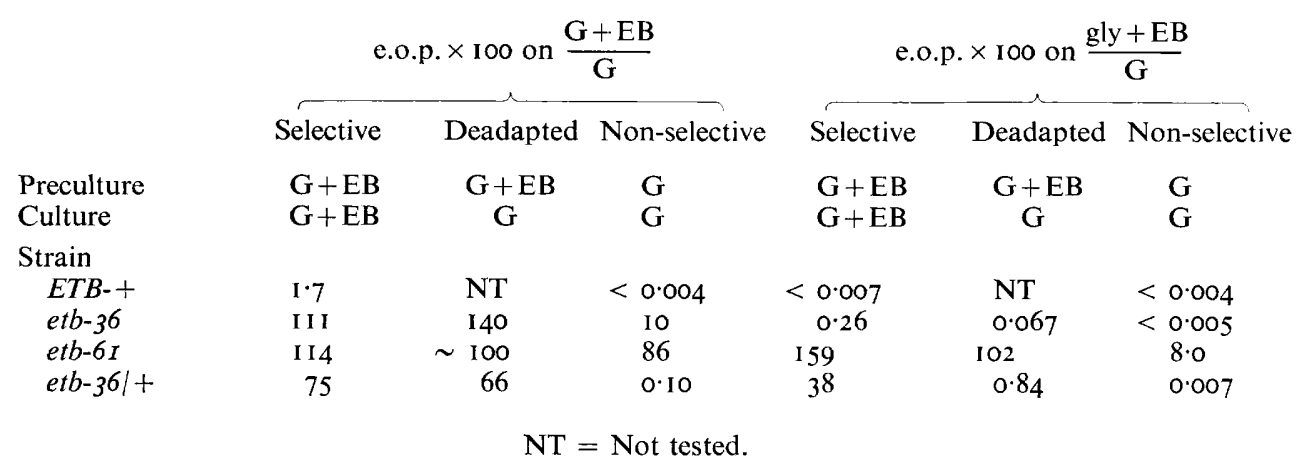

broth. Fig. 5 and 6 show that: (i) the wild-type is sensitive to EB, although the culture turbidity does increase several-fold before growth arrest; (ii) mutants isolated either on $\mathrm{G}+\mathrm{EB}$ or on GLY + EB are both resistant to EB in liquid media whether the carbon source is G or GLY; (iii) both mutants show a long lag period after dilution from non-selective to selective medium; (iv) there was no significant lag if mutants were pregrown in selective medium; (v) $e t b-6 I$ has a shorter lag period and faster growth rate in EB media than $e t b-36$; (vi) the lag period in GLY + EB broth is longer for both mutants than G+EB broth; (vii) the heterozygous diploid $e t b-36 /+$ is initially sensitive but gives rise to resistant $e t b$ 36/etb-36 mitotic recombinants as shown by the sample plating experiment below.

Distinction between EB adaptation or genetic selection. To determine whether the lag observed in Fig. 5 and 6 is due to adaptation to selective conditions or selection of genetic variants better able to grow in the presence of $\mathrm{EB}$, cultures from $\mathrm{G}+\mathrm{EB}$ broth were subcultured in $\mathrm{G}$ broth twice and then the e.o.p. of these 'deadapted' cells was compared to cells from selective or non-selective cultures. It is clear from the results in Table 2 that the wildtype is sensitive on both G+EB and GLY + EB plates and mutant $e t b-36$ and hybrid $e t b-36 /+$ are sensitive only on GLY + EB plates confirming previous observations. Again it was found that on $\mathrm{G}+\mathrm{EB}$ plates $e t b-36 /+$ gives rise to a small percentage of resistant colonies even though this heterozygote is sensitive. The increase in e.o.p. of $e t b-36$ on $\mathrm{G}+\mathrm{EB}$ to $\mathrm{I} 00 \%$ after pregrowth in $\mathrm{G}+\mathrm{EB}$ medium was retained even after selective cultures were 'deadapted' therefore these cultures probably contain variants of the original mutant strain better able to grow in the presence of EB. The high e.o.p. of eth-36/+ on G+EB of the $\mathrm{G}+\mathrm{EB}$ culture indicates that it now contains diploid segregants of genotype $e t b-36 / e t b-36$. Selective cultures from G+EB broth showed a decrease in e.o.p. on GLY + EB plates after 
about 13 generations of non-selective growth for mutant $e t b-36$ and its diploid hybrid. For these two cultures 'deadaptation' had some significance again indicating differences between the effect of EB on G as opposed to GLY medium.

\section{DISCUSSION}

Following a rigorous search for petites in $H w$ it can be concluded that this type of mitochondrial mutant cannot be isolated in this yeast. No petites were obtained after cells of $H w$ were treated with either u.v. or EB under conditions which would have produced up to $100 \%$ petite mutagenesis in $S c$. The mutant isolation methods used in this search for petites in $\mathrm{Hw}$ were sensitive enough to detect a very rare glycerol-negative mutant which was shown not to be a petite (Crandall, 1973). Petite-negativity in $H w$ is correlated with the lack of glucose repression and the lack of fermentation ability as reported for other obligately aerobic yeasts (Bulder, I 964 $a, b$; De Deken, I $966 a, b$; McClary \& Bowers, I967; Kellerman et al. 1969; Mahler \& Perlman, 197I).

Although EB does not produce petite mutants in $H w$ it does inhibit growth. Cells of the wild-type are sensitive to EB on fermentable as well as non-fermentable substrates. Growth inhibition of single cells on plates is immediate (no microcolonies develop), whereas in liquid one or more divisions occur before final growth arrest. When cells of $H w$ were treated with EB under non-growing conditions no lethality and no mutagenesis occurred. The only real evidence of lethality obtained with $H w$ was under growing conditions using very high concentrations of $\mathrm{EB}$ ( $100 \mu \mathrm{g} \mathrm{EB} / \mathrm{ml}$ for $7.5 \mathrm{~h}$ ). This concentration is 5 times the concentration necessary to inhibit growth in $H w$ and almost 100 times the concentration and roo times the length of time necessary to cause the petite mutation in $S c$. Whether the loss of viability represents a lethal petite mutation or simply loss of viability during aeration in the presence of a growth inhibitor remains to be determined. Lethality caused by acriflavin or EB has been reported in other petite-negative yeasts (Bulder, I964a, b; Mahler \& Perlman, I97I).

Although EB does not mutate cells it does have a different inhibitory effect depending on the carbon source. GLY + EB medium is more selective than G+EB medium as judged by several criteria: (i) more than one mutation is required for growth on GLY +EB since the mutation frequency is $\sim \mathrm{IO}^{-8}$, whereas the mutation frequency to growth on $\mathrm{G}+\mathrm{EB}$ is $\sim \mathrm{IO}^{-6}$; (ii) mutants resistant on $\mathrm{G}+\mathrm{EB}$ are still sensitive on GLY +EB plates, whereas mutants resistant on GLY + EB and sensitive revertants are resistant on $\mathrm{G}+\mathrm{EB}$; (iii) less than $4 \mu \mathrm{g} \mathrm{EB} / \mathrm{ml}$ will inhibit growth on GLY, whereas $20 \mu \mathrm{g} \mathrm{EB} / \mathrm{ml}$ are required for inhibition of growth on G; (iv) EB treatment of cells reversibly inhibits their growth on nonfermentable substrates such as GLY, whereas no growth inhibition is observed if EBtreated cells are plated on $\mathrm{G}$; (v) longer lags are observed before resistant cells grow in $\mathrm{GLY}+\mathrm{EB}$ broth or on GLY + EB plates than in G + EB media; (vi) cells adapted for growth in $\mathrm{G}+\mathrm{EB}$ broth and then 'deadapted' by I3 generations of growth in non-selective broth maintain a high e.o.p. on G+EB plates but lose the ability to grow on GLY +EB plates suggesting an inducible EB resistance function on GLY.

Mutant $e t b-36$ was analysed genetically (Crandall \& Richter, to be published) and had resulted from recessive mutations at two linked nuclear loci called $e t b l$ and $e t b 2$. These two loci, together with the gene controlling resistance on GLY $+E B$, probably control gene products involved in mitochondrial sites sensitive to EB. Biochemical studies to characterize these mutations are currently underway.

An EB resistant mutant has been isolated in Sc (J. C. Mounolou, personal communication; see Table I) which is not a petite since it is capable of growth on GLY. Thus this 
mutant must be altered at the site sensitive to EB petite mutagenesis. It must also be resistant at the three sites identified in $H w$ affecting growth on GLY in the presence of EB. Further biochemical and genetic analyses of the EB-resistant mutants isolated in $S c$ and $H w$ are required before the interrelationships between the genes for petite production, EB resistance and glucose repression may be understood.

M. C. expresses special thanks to B. Dujon for many helpful explanations. Professor H. R. Mahler is thanked for suggesting the glucose repression experiment. This investigation was performed while M. C. was a postdoctoral fellow of the newly established exchange fellowship programme between the U.S. National Science Foundation and the French Centre National de la Recherche Scientifique. The paper was written while M. C. was being supported by U.S.P.H.S. Grant GM I 8708.

\section{REFERENCES}

Bolotin, M., Coen, D., Deutsch, J., Dujon, B., Netter, P., Petrochilo, E. \& Slonimski, P. P. (i971). La récombinaison des mitochondries chez Saccharomyces cerevisiae. Bulletin de l'Institut Pasteur 69 , $215-239$.

Brock, T. D. (196I). Physiology of the conjugation process in the yeast Hansenula wingei. Journal of General Microbiology 26, 487-497.

Bulder, C. J. E. A. (I964a). Induction of petite mutation and inhibition of synthesis of respiratory enzymes in various yeasts. Antonie van Leeuwenhoek. Journal of Microbiology and Serology 30, 1-9.

BUlder, C. J. E. A. $(1964 b)$. Lethality of the petite mutation in petite negative yeasts. Antonie van Leeuwenhoek. Journal of Microbiology and Serology 30, 442-454.

Coen, D., Deutsch, J., Netter, P., Petrochilo, E. \& Slonimski, P. P. (1970). Mitochondrial genetics. I. Methodology and Phenomenology. In Control of Organelle Development, pp. 449-496. Symposium of the Society for Experimental Biology. London: Cambridge University Press.

Conti, S. F. \& Brock, T. D. (1965). Electron microscopy of the cell fusion in conjugating Hansenula wingei. Journal of Bacteriology 90, 524-533.

Crandall, M. (1973). A respiratory-deficient mutant in the obligately aerobic yeast Hansenula wingei. Journal of General Microbiology 75, 377-38r.

Crandall, M. \& Brock, T. D. (I968). Molecular basis of mating in the yeast Hansenula wingei. Bacteriological Reviews 32, $139-163$.

De Deken, R. H. (I966a). The Crabtree effect : a regulatory system in yeast. Journal of General Microbiology 44, I49-156.

De Deken, R. H. (1966b). The Crabtree effect and its relation to the petite mutation. Journal of General Microbiology 44, I 57-165.

Ephrussi, B. \& HotTinguer, H. (I95I). Cytoplasmic constituents of heredity. On an unstable cell state in yeast. Cold Spring Harbor Symposium of Quantitative Biology 16, 75-85.

Goldring, E. S., Grossman, L. I. \& Marmur, J. (I97I). Petite mutation in yeast. II. Isolation of mutants containing mitochondrial deoxyribonucleic acid of reduced size. Journal of Bacteriology 107, 377-381.

Kellerman, G. M., Biggs, D. R. \& Linnane, A. W. (1969). Biogenesis of mitochondria. XI. A comparison of the effect of growth-limiting oxygen tension, intercalating agents, and antibiotics on the obligate aerobe Candida parapsilosis. Journal of Cell Biology 42, 378-391.

Luha, A., Sacroe, L. E. \& Whittaker, P. A. (I971). Biosynthesis of yeast mitochondria. Drug effects on the petive negative yeast Kluyveromyces lactis. Biochemical and Biophysical Research Communications 44, 396-402.

MCClary, D. O. \& Bowers, W. D. (1967). Structural differentiation of obligately aerobic and facultatively anaerobic yeasts. Journal of Cell Biology, 32, 519-524.

Mahler, H. R. \& Perlman, P. S. (I97I). Mitochondriogenesis analysed by blocks on mitochondrial translation and transcription. Biochemistry 10, 2979-2989.

Mahler, H. R. \& Perlman, P. S. (1972). Effects of mutagenic treatment by ethidium bromide on cellular and mitochondrial phenotype. Archives of Biochemistry and Biophysics 148, I I5-129.

Nagley, P. \& Linnane, A. W. (1970). Mitochondrial DNA deficient petite mutants of yeast. Biochemical and Biophysical Research Communications 39, 989-996. 
Perlman, P. S. \& Mahler, H. R. (I97I $a$ ). A premutational state induced in yeast by ethidium bromide. Biochemical and Biophysical Research Communications 44, 26r-267.

Perlman, P. S. \& Mahler, H. R. (197I $b$ ). Molecular consequences of ethidium bromide mutagenesis Nature, London 23I, I2-I6.

Preer, J. R., JUN. (I97I). Extrachromosomal inheritance: hereditary symbiants, mitochondria, chloroplasts Annual Review of Genetics 5, 36I-406.

Slonimski, P. P. (1956). Adaptation respiratoire: développement du système hémoproteique induit par l'oxygène Proceedings of the 3rd International Congress of Biochemistry, pp. 242-252. New York: Academic Press.

Slonimski, P. P., Perrodin, G. \& Croft, J. H. (I968). Ethidium bromide induced mutation of yeast mitochondria: complete transformation of cells into respiratory deficient non-chromosomal 'petites'. Biochemical and Biophysical Research Communications 30, 232-239.

Weislogel, P. O. \& Butow, R. A. (I97I). Control of the mitochondrial genome in Saccharomyces cerevisiae. The fate of mitochondrial membrane proteins and mitochondrial deoxyribonucleic acid during petite induction. Journal of Biological Chemistry 246, 5I I 3-5 I I9.

Wickerham, L. J. (1970). Hansenula H. et P. Sydow. In The Yeasts: A Taxonomic Study, pp. 226-3I5. Edited by J. Lodder. Amsterdam: North-Holland Publishing Co. 